PROCEEDINGS OF THE

AMERICAN MATHEMATICAL SOCIETY

Volume 140, Number 12, December 2012, Pages 4205-4208

S 0002-9939(2012)11267-5

Article electronically published on April 4, 2012

\title{
A FATOU-BIEBERBACH DOMAIN INTERSECTING THE PLANE IN THE UNIT DISK
}

\author{
ERLEND FORNÆSS WOLD
}

(Communicated by Franc Forstneric)

\begin{abstract}
We construct a Fatou-Bieberbach domain $\Omega$ in $\mathbb{C}^{2}=\mathbb{C}_{z} \times \mathbb{C}_{w}$ such that one of the connected components of $\Omega \cap \mathbb{C}_{z}$ is the unit disk $\mathbb{D}_{z} \subset \mathbb{C}_{z}$.
\end{abstract}

\section{INTRODUCTION}

It was asked by Rosay and Rudin 8 , whether the intersection of a FatouBieberbach domain and a complex line in $\mathbb{C}$ could be a circular disk. We will show the following:

Theorem 1.1. There exists a Fatou-Bieberbach domain $\Omega \subset \mathbb{C}^{2}$ with $\mathbb{D}_{z} \subset \mathbb{C}_{z} \cap \Omega$ and $b \mathbb{D}_{z} \subset b \Omega$.

A Fatou-Bieberbach domain in $\mathbb{C}^{2}$ is, by definition, a proper subdomain of $\mathbb{C}^{2}$ which is biholomorphic to $\mathbb{C}^{2}$. The theorem is related to the problem of embedding Riemann surfaces properly into $\mathbb{C}^{2}$; it follows immediately that the unit disk can be realized as a closed submanifold of $\mathbb{C}^{2}$. Indeed, the first proof of the fact that the unit disk admits a proper holomorphic embedding in $\mathbb{C}^{2}$ goes as follows: Start with a Fatou-Bieberbach domain $\Omega \subset \mathbb{C}^{2}$ with the Runge property (for the existence, see e.g. [8]). There must exist a complex line $L$ in $\mathbb{C}^{2}$ such that $L_{\Omega}:=\Omega \cap L \neq L$. By the Runge property, each connected component of $L_{\Omega}$ is simply connected, and by the Riemann mapping theorem, they are all conformally equivalent to the unit disk.

Similarly, it follows from Theorem 1.4 below that the same holds for any bordered Riemann surface, a fact that was proved in [6]. In fact, most of the approaches to the open problem of embedding Riemann surfaces properly into $\mathbb{C}^{2}$ amounts to circumventing having to prove Theorem 1.1. One proves instead that there is another surface, conformally equivalent to $\mathbb{D}_{z}$ (resp. $\mathcal{R}$ ), for which the theorem holds.

J. Globevnik considered the same problem in 7, where he showed that the intersection could be as close to the disk in the $\mathcal{C}^{1}$-norm as desired. So in that sense our result is much stronger. On the other hand, in his example there are no other connected components of $\Omega \cap \mathbb{C}_{z}$ than the (topological) disk.

Received by the editors May 18, 2011 and, in revised form, May 20, 2011.

2010 Mathematics Subject Classification. Primary 32E30, 32H02.

(C)2012 American Mathematical Society 
By the standard construction of Fatou-Bieberbach domains using the push-out method, proving the following is the key issue:

Theorem 1.2. Let $K \subset \mathbb{C}^{2}$ be a polynomially convex compact set with $K \cap \mathbb{D}_{z} \Subset \mathbb{D}_{z}$, and let $R, \epsilon>0$. Then there exists $\phi \in A u t_{h o l} \mathbb{C}^{2}$ such that $\|\phi-i d\|_{K}<\epsilon$ and $\phi\left(b \mathbb{D}_{z}\right) \subset \mathbb{C}^{2} \backslash R \cdot \overline{\mathbb{B}^{2}}$.

Inductively one constructs $\phi_{j} \in A u t_{h o l} \mathbb{C}^{2}$ such that $\phi(k)\left(b \mathbb{D}_{z}\right):=\phi_{k} \circ \cdots \circ$ $\phi_{1}\left(b \mathbb{D}_{z}\right) \subset \mathbb{C}^{2} \backslash k \cdot \overline{\mathbb{B}^{2}}$, making sure that the sequence $\phi(k)$ converges uniformly on compact subsets of $\mathbb{D}_{z}$ and that the inverse sequence $\phi(k)^{-1}$ converges uniformly on compact subsets of $\mathbb{C}^{2}$. The domain $\Omega$ of convergence of $\phi(k)$ will be biholomorphic to $\mathbb{C}^{2}$ (see e.g. [3] and [8]).

Using Theorem 1.1 and Lemma 2.2 in [5], it suffices to show the following:

Lemma 1.3. Let $K \subset \mathbb{C}^{2}$ be a polynomially convex compact set and assume that $K \cap \mathbb{D}_{z} \Subset \mathbb{D}_{z}$. Then for $\epsilon, R>0$ there exists a neighborhood $\Omega$ of $K \cup \overline{\mathbb{D}_{z}}$ and an isotopy of injective holomorphic maps $\phi:[0,1] \times \Omega \rightarrow \mathbb{C}^{2}$ such that the following hold:

a) $\phi_{0}=i d$,

b) $\left\|\phi_{t}-i d\right\|_{K}<\epsilon$ for all $t \in[0,1]$,

c) $\phi_{t}\left(K \cup \overline{\mathbb{D}_{z}}\right)$ is polynomially convex for all $t \in[0,1]$, and

d) $\phi_{1}\left(b \mathbb{D}_{z}\right) \subset \mathbb{C}^{2} \backslash R \cdot \mathbb{B}^{2}$.

The difficulty in proving such a lemma comes from the fact that $b \mathbb{D}_{z}$ is not polynomially convex.

In this paper we will only give a proof of Theorem 1.1, but by employing parameter versions of the technique of exposing points from [6] together with additional Cartan gluing due to Forstneric [4, it is possible to show the following:

Theorem 1.4. Let $\overline{\mathcal{R}} \subset \mathbb{C}^{2}$ be a polynomially convex bordered Riemann surface. There exists a Fatou-Bieberbach domain $\Omega \subset \mathbb{C}^{2}$ with $\mathcal{R} \subset \Omega$ and $b \mathcal{R} \subset b \Omega$.

We will include a brief sketch of the main technical part of the proof after Lemma 2.1 in the next section. For instance, we could let $\mathcal{R}$ be a small perturbation in $\mathbb{C}^{2}$ of an annulus in $\mathbb{C}_{z}$ such that the perturbation is polynomially convex.

Question 1.5. Does there exist a Fatou-Bieberbach domain $\Omega$ in $\mathbb{C}^{2}$ intersecting the complex plane in an annulus? Such a domain $\Omega$ could not be rationally convex.

Recall that a complex manifold $X$ is said to have the density property (resp. volume density property) if the Lie algebra generated by the complete holomorphic vector fields on $X$ is dense in the algebra of entire vector fields (resp. divergence free vector fields). The proof of our main theorem depends crucially on the fundamental fact that $\mathbb{C}^{2}$ has the density property (see [1, 2]). A more trivial but equally crucial ingredient is the existence of so-called shear automorphisms of $\mathbb{C}^{2}$ (see e.g. 8]). The following is therefore an interesting (and supposedly very hard) question:

Question 1.6. Does Theorem 1.2 hold if we replace $\mathbb{C}^{2}$ by a manifold $X$ with the density or volume density property? 


\section{Proof of Theorem 1.1}

Let $\pi_{1}$ denote the projection onto the first coordinate $\mathbb{C}^{2} \rightarrow \mathbb{C}_{z}$. By a $\pi_{1}$-exposed point for an embedding $\varphi\left(\overline{\mathbb{D}_{z}}\right)$ of the unit disk, we will mean a point $\varphi(a), a \in b \mathbb{D}_{z}$, with the property that $\pi \circ \varphi$ is an embedding near $a$, and

$$
\pi_{1}^{-1}(\pi(\varphi(a))) \cap \varphi\left(\overline{\mathbb{D}_{z}}\right)=\varphi(a) .
$$

Lemma 2.1. Let $K \subset \mathbb{C}^{2}$ be a polynomially convex compact set and assume that $K \cap \mathbb{D}_{z} \Subset \mathbb{D}_{z}$. Then for $\epsilon, R>0$ there exists a point $a \in b \mathbb{D}_{z}$ and an isotopy $\varphi_{t} \in A_{u t} t_{h o l} \mathbb{C}^{2}$ such that the following hold:

a) $\varphi_{0}=i d$,

b) $\left\|\varphi_{t}-i d\right\|_{K}<\epsilon$ for all $t \in[0,1]$, and

c) $\varphi_{1}(a)$ is $\pi_{1}$-exposed with $\left|\pi_{1}\left(\varphi_{1}(a)\right)\right| \geq R$.

Proof. Pick a point $\tilde{a} \in b \mathbb{D}_{z}$. Since $\mathbb{C}^{2}$ has the density property, there exists an isotopy $\varphi_{t} \in A u t_{h o l} \mathbb{C}^{2}$ such that a) and b) are satisfied and $\left|\pi_{1}\left(\varphi_{1}(\tilde{a})\right)\right| \geq R$. To prove it, define an isotopy of injective holomorphic maps near $K \cup\{\tilde{a}\}$, being the identity near $K$ and moving $\tilde{a}$ to a suitable point away from $K$, and then apply Theorem 1.1 in [5] (approximating using the Andersén-Lempert approach gives an isotopy of holomorphic automorphisms staying close to the identity near $K$ ). Let $a_{1}, \ldots, a_{m}$ be the points in $b \mathbb{D}_{z}$ where $\pi_{1} \circ \varphi_{1}$ achieves its maximum. By an arbitrarily small perturbation of $\varphi$ we may assume that one of these points is $\pi_{1}$-exposed.

Remark 2.2. To prove Theorem 1.4 we need to prove the corresponding lemma for a bordered Riemann surface $\mathcal{R}$ in $\mathbb{C}^{2}$. In that case, there are finitely many boundary components $\Gamma_{j}$ of $b \mathcal{R}$, and we want to simultaneously expose points $a_{j} \in \Gamma_{j}$ by an isotopy $\varphi_{t}$ of injective holomorphic maps defined near $K \cup \overline{\mathcal{R}}$. The first step would be to "thicken" $\overline{\mathcal{R}}$ using an embedding of a neighborhood of the zero section of the normal bundle of $\overline{\mathcal{R}}$. Exposing points of this thickened $\overline{\mathcal{R}}$ is achieved by a parametrized version of the technique of exposing points from [6]. Making sure that the isotopy is close to the identity near $K \cap \overline{\mathcal{R}}$, this isotopy may be approximately glued to the identity near $K$ by a Cartan gluing technique due to Forstnerič 4 .

Proof of Lemma 1.3. Choose a slightly bigger compact $K^{\prime}$ such that $K \subset\left(K^{\prime}\right)^{\circ}$. Choose first an isotopy $\varphi_{t}$ as in Lemma[2.1 with $R$ chosen such that $\pi_{1}\left(K^{\prime}\right) \subset R \cdot \mathbb{D}_{z}$. Set $b:=\pi_{1}\left(\varphi_{1}(a)\right)$.

For any small $\delta>0$ set $\psi_{t}^{\delta}(z, w):=\left(z, w+\frac{t \delta}{z-b}\right)$. By Lemma 2.1 in 9 ] there exists an isotopy $\Theta_{t}^{\delta} \in A u t_{h o l} \mathbb{C}^{2}$ such that the following hold:

i) $\Theta_{0}^{\delta}=i d$,

ii) $\left\|\Theta_{t}^{\delta}-i d\right\|_{\psi_{1}^{\delta}\left(\varphi_{1}\left(K^{\prime}\right)\right)}<\epsilon^{\prime}$, and

iii) $\Theta_{1}^{\delta} \circ \psi_{1}^{\delta} \circ \varphi_{1}\left(b \mathbb{D}_{z} \backslash\{a\}\right) \subset \mathbb{C}^{2} \backslash R \cdot \overline{\mathbb{B}^{2}}$.

We remark that it is not stated in [9] that $\Theta$ can be chosen to be an isotopy, but by the way it is constructed, using the Andersén-Lempert theory and precomposing with a shear map, it is clear that this can be achieved.

Finally let $\sigma_{t}^{\mu} \in A u t_{h o l} \mathbb{C}^{2}$ be defined by $\sigma_{t}^{\mu}(z, w):=((1-t \mu) \cdot z, w)$ for $0<\mu<1$. 
Choose $\delta$ and $\mu$ very small, and define a piecewise smooth isotopy $\phi_{t}$ by first flowing along $\sigma_{t}^{\mu}$ from time 0 to 1 , then $\varphi_{t}$ from 0 to 1 , then $\psi_{t}^{\delta}$ from 0 to 1 , and finally $\Theta_{t}$ for 0 to 1 . It is clear that a), b) and d) will hold if $\delta$ and $\mu$ are small enough. Note that if $\phi_{t}$ is close enough to the identity on $K^{\prime}$, then $\phi_{t}(K)$ will be polynomially convex. It is well known that the polynomial hull of $\phi_{t}(K) \cup \phi_{t}\left(b \mathbb{D}_{z}\right)$ is a variety in the complement of $\phi_{t}(K) \cup \phi_{t}\left(b \mathbb{D}_{z}\right)$, so we also have c).

\section{REFERENCES}

1. Andersén, E.; Volume-preserving automorphisms of $\mathbb{C}^{n}$. Complex Variables Theory Appl. 14 (1990), no. 1-4, 223-235. MR 1048723 (91d:32047)

2. Andersén, E. and Lempert, L.; On the group of holomorphic automorphisms of $\mathbb{C}^{n}$. Invent. Math. 110 (1992), no. 2, 371-388. MR.1185588 (93i:32038)

3. Dixon, P. G. and Esterle, J.; Michael's problem and the Poincaré-Fatou-Bieberbach phenomenon. Bull. Amer. Math. Soc. (N.S.) 15 (1986), no. 2, 127-187. MR854551 (88d:46090)

4. Forstnerič, F.; Noncritical holomorphic functions on Stein manifolds. Acta Math. 191 (2003), no. 2, 143-189. MR 2051397 (2005b:32021)

5. Forstnerič, F. and Rosay, J.-P.; Approximation of biholomorphic mappings by automorphisms of $\mathbb{C}^{n}$. Invent. Math. 112 (1993), no. 2, 323-349. MR.1213106 (94f:32032)

6. Forstnerič, F. and Wold, E. F.; Bordered Riemann surfaces in $\mathbb{C}^{2}$. J. Math. Pures Appl. (9) 91 (2009), no. 1, 100-114. MR2487902 (2010b:32008)

7. Globevnik, J.; On Fatou-Bieberbach domains. Math. Z. 229 (1998), no. 1, 91-106. MR 1649310 (99g:32050)

8. Rosay, J.-P. and Rudin, W.; Holomorphic maps from $\mathbb{C}^{n}$ to $\mathbb{C}^{n}$. Trans. Amer. Math. Soc. 310 (1988), no. 1, 47-86. MR929658 (89d:32058)

9. Wold, E. F.; Embedding Riemann surfaces properly into $\mathbb{C}^{2}$. Internat. J. Math. 17 (2006), no. 8, 963-974. MR2261643 (2007h:32027)

Matematisk Institutt, Universitetet i Oslo, Postboks 1053 Blindern, 0316 Oslo, NoRWAY

E-mail address: erlendfw@math.uio.no 